\title{
Comprehension of prenominal adjective orders
}

\author{
JOSEPH H. DANKS and MARY ANN SCHWENK \\ Kent State University, Kent, Ohio 44242
}

\begin{abstract}
In two experiments, phrases describing a referent object contained two prenominal adjectives in either normal or inverted order. The time to identify the position of the referent in a display was a function of both the adjective order and the nonreferent context. If the referent appeared with a nonreferent differing from it only in size or number. the normal order of adjectives facilitated responding. However, if the referent appeared with a nonreferent differing from it only in color, the inverted order of adjectives resulted in faster identification times. These results support a pragmatic communication rule that. when the more discriminating adjectives are ordered earlier in a series. comprehension is facilitated.
\end{abstract}

One of the most popular strategies of contemporary psycholinguistics has been to interpret various laboratory phenomena in terms of constructs derived from linguistic theory. Inasmuch as contemporary linguistics has been dominated by a concern for syntactic problems. the interpretation of psychological experiments was likewise syntactically oriented. Recently, however, psychologists have become disillusioned with such a strategy, because of both the swiftly changing linguistic theory and the lack of success in restricting interpretation of behavioral phenomena to syntactic variables. A case in point is the ordering of prenominal adjectives in English. Speakers agree as to which orders are grammatical and which are not, for example, the two large red cars and the red large two cars, respectively (Danks \& Glucksberg, 1971: Martin, $1969 \mathrm{~b}$ ). The order is apparently determined by a formal syntactic rule, but linguists have been unsuccessful in deriving such a rule without ad hoc assumptions, for example. Vendler (1968). Hence, psychologists have considered other types of rules.

Martin (1969b) and Danks and Glucksberg (1971) proposed a semantic rule for explaining adjective ordering that was based on the qualities referred to by the adjectives-definiteness, absoluteness, or intrinsicalness. For example, in comparison to size, color is more definite in meaning, changes less from object to object (absoluteness). and is considered a more intrinsic property of the object. Red refers to a certain range of wavelengths, independent of whether the object is a car or a button; but a large car is a very different size than a large button. Martin (1969a, b) further complicated the model by suggesting accessibility as a mediating mechanism. The semantic dimensions of definiteness and absoluteness determine the accessibility of adjectives, which in turn determine their ordering. Martin operationally defined accessibility in terms of response latency to provide an adjectival description of a physical stimulus. He found that the response speed was correlated with ordering: The nearer an adjective was preferred to the noun, the more accessible it was. However, speakers may store their adjectives such that those associated with frequently informative dimensions are most accessible. In fact, Martin (1969b) found that Thorndike-Lorge frequency correlated significantly with preferred adjective order, but in the opposite direction an accessibility notion would predict. The more frequent adjectives were preferred further from the noun than were the less frequent ones.

This relation between frequency and adjective ordering can be predicted, however, through the introduction of pragmatic factors. Following a suggestion by Danks and Glucksberg (1971), Danks and Schwenk proposed a pragmatic communication rule to account for the influence of communication context on preferred adjective ordering, namely, "the more discriminating adjectives tend to be placed first in a series of adjectives [1972, p. 186]." For example, when comparing two large cars, one red and one blue, one might very well say the red large car to emphasize the criterial property for the listener. This rule states that the elements of physical context in which a referent occurs influence adjective ordering. It goes beyond but yet incorporates the semantic rule for adjective ordering, which considers the properties denoted by the adjectives as the only factors in the ordering process. Both the properties denoted by the adjective and the communication context are important determinants of adjective ordering according to the pragmatic communication rule. If one makes the not unreasonable assumption that the more frequently used adjectives refer to properties more frequently used to discriminate referents, then this pragmatic communication rule accounts for the frequency relation.

Danks and Schwenk (1972) tested the pragmatic communication rule by instructing Ss to select which of two phrases better described a referent object appearing in different physical contexts. The criterion for the Ss' decision was the phrase that would more likely insure that a listener would make a correct identification of a referent object. One phrase described the referent with the adjectives in normal order, the other phrase used an inverted order. When the referent was presented in a context of a nonreferent object that differed from it solely on the basis of color, the phrase containing an inverted order of adjectives was preferred. When size was 
the dimension on which the referent and nonreferent differed. the normal adjective order was preferred even more strongly than in a control condition, where neither adjective was needed to discriminate the referent from the nonreferent. These results parallel those obtained by Oller and Sales (1969). Although arriving at similar conclusions. they admit the lack of rigid experimental coutrols in their demonstrations. Hence, the preferred ordering of prenominal adjectives can be manipulated, at least in part, by nonlinguistic factors, such as the physical context surrounding the referent.

Does changing of adjective order ease the identification task for the listener or is inversion a nonfunctional act by the speaker? Since it is unlikely that a behavior as common as talking would develop a significant nonfunctional component, we predicted that listeners would be able to make icientifications faster given an adjective order appropriate to the context. The Danks and Schwenk (1972) experiment was considered to be a preferential production task for speakers. The current experiments were designed as the comprehension analogue for listeners. If color is the sole discriminating at tribute in a set of objects, then listeners should be able to comprehend a description and make an identification of the described object faster with an inverted order of adjectives in the description than with a normal order. If size is the criterial attribute. then the normal order should facilitate identification. Thus. if contextual comparisons are important. congruence between adjective order and physical context should facilitate responding.

\section{EXPERIMENT I}

\section{Method}

\section{Subjects}

Twenty male undergraduates at Kent State Cniversity participated in the experiment to fulfill partially a requirement in a general psychology course. All Ss were native English speakers and naive to this experimental task.

\section{Vaterials}

Colored line drawings of 10 different objects-a car. pencil. kes clock. button. fish. hammer. scissors, and a table-were prepared. The drawings were the same ones used by Danks and Schwenk (1972). Each object was drawn in two different sizes and two different colors. resulting in four unique versions of each object.

Slides containing four drawings, one in each quadrant of the slide. were composed from the total set of 80 drawings. There were five different slide types. three experimental types and two fillers. All experimental slides depicted two versions of the same object plus two filler objects. If the two versions of the same object were the sami color but different in size. the slide was termed size relevant. since size was the dimension on which the two objects could be discriminated. If the two versions of the same object differed only in color. the slides were termed color relevant. since color was the discriminative dimension. Slides containing two objects tiat were the same bet differed in both size and color were called either rekvant. because either dimenvion was retevant and wficion fo identity lis referent.
The two filler objects on each experimental slide were selected arbitrarily with two restrictions: On each slide four different colors were present (except for size, when only three were present) and two large and two small drawings were present. Filler slides were termed both relevant and neither relevant. Since both-relevant slides consisted of all four versions of a single object differing in color and size, both adjectives were needed to discriminate between objects. Neither adjective was needed to discriminate between objects in the neither-relevant slides. because they consisted of four different objects, so that the object name alone was sufficient to identify an object. There were nine each of the experimental slides and 27 each of the two filler types.

A criterion object (the referent) was selected on each slide. For the experimental slides, it was one of the two versions of the same object. For the filler slides. it was arbitrarily selected. The referent appeared equally of ten in each quadrant for each slide type. On the experimental slides, the nonreferent contrasting with the referent occurred equally often in the other three quadrants.

The verbal material was a tape recorded description of the referent. consisting of a determiner. two adjectives, and a noun. $\mathrm{Hal}^{-}$of the descriptions for each slide type presented the adjectives in normal order (size then color) and the other half presented the adjectives in an inverted order, e.g., the large red car and the green small pencil, respectively. Intonation of the descriptive phrase was varied between Ss. Half of the Ss heard a description with a stress intonation for all phrases. It consisted of heavy stress on the first adjective. followed by a juncture and light stress on the second adjective and noun. The other half heard a list intonation consisting of moderate stress on both adjectives. falling intonation on the noun, and junctures after both adjectives.

The slides and phrases were arbitrarily ordered for presentation according to the following restrictions: The same object in any of its versions could not appear as a referent in succession: the referent could not be in the same quadrant nor the same color more than twice in succession, nor the same size three times in succession; more than two slides of the same type could not occur together; and no more than three normal or three inverted orders could occur successively. Following nine warm-up trials, one of each of the experimental slides and three of each of the fillers. there were four blocks of 18 slides containing two of each of the experimental slides and six of each of the fillers. One normal and one inverted order of adjectives occurred with each experimental slide in each block.

\section{Procedure}

All Ss were tested individually. Ss were told that they would view a series of slides. Shortly after the slide appeared. they would hear a description of one of the four objects on the slide. They were to orally identify the quadrant containing the described object as quickly as possible without making errors. To provide them with appropriate labels. a sheet of paper divided into four quadrants labeled A. B. C, and D was placed in front of them. Ss could refer to the sheet in making their responses.

The slides were presented at about 10 -sec intervals. One second after the slide appeared, the $S$ heard the description over earphones. A clock (accurate to $.01 \mathrm{sec}$ ) was started by a signal on the tape (inaudible to the $S$. The signal coincided with the first word in the description. the. The clock was stopped by the $\mathrm{S}$ 's response activating a voice key. The $\mathrm{E}$ recorded the response. the latency, and noted an! false triggerings of the voice key. The entire procedure. including instruction. took about $20 \mathrm{~min}$

\section{Results}

There were only 11 errors out of 480 experimental trials. These errors were evenly distributed across 
conditions. The mean latency for correct responses was computed for each $\mathbf{S}$ on each experimental trial type. These scores were analyzed by a 2 by 2 by 3 analysis of variance. Intonation (stress or list) was a between-S variable. The within-S variables were dimension relevant (color, size, or either) and adjective order (normal or inverted). There was no main effect of intonation nor of any of its interactions; hence, it is not discussed further.

The remaining effects were all significant: adjective order $[F(1,18)=60.51, p<.001]$, dimension relevant $[F(2,36)=6.04, \quad p<.01]$, and their interaction $[F(2.36)=4.77, p<.02]$. The relevant means of these effects are presented in Table 1 . In all three dimension relevant conditions, the inverted order produced significantly faster identifications than the normal order of adjectives. The critical comparison for this experiment was the interaction of color and size dimensions relevant with normal and inverted adjective orders. This interaction was tested both with Newman-Keuls comparison (Winer, 1971) and with a separate analysis of variance. It was significant in both cases $[q(2,36)=5.66, \quad p<.01$ and $F(1,18)=8.35$, $\mathrm{p}<.01]$. Hence, the main prediction was supported: The inverted order of adjectives facilitated identification of the object in the color relevant context more than in the size relevant context.

However, there was a discrepancy in these data. With both size and either relevant conditions, Ss responded significantly faster with the inverted order than with the normal order. Just the opposite had been predicted: When size was relevant or when either adjective was relevant, the normal order of adjectives should have produced faster identification. There were two possible explanations of this result. First, the color adjective always carried more information than did the size adjective. In every slide, there were four different colors present (except for size relevant) but there were only two sizes present. In most cases, as soon as the $\mathrm{S}$ heard the color he could respond correctly, and color was first in the inverted order. If $S$ heard the size adjective first (normal order), he always had to wait for more information. Hence, there was a bias in favor of the inverted order. Second, the colors of the objects were subjectively more salient than the sizes. The colors were brighter and easier to identify than were the sizes. The sizes could be established only by comparison, although the $\mathrm{S}$ soon learned which size was which. Therefore, Ss may have tested the color dimension earlier or faster than size.

\section{EXPERIMENT II}

Experiment II was designed to eliminate the discrepancies between the color and size properties. The size dimension was replaced with a quantity dimension. There could be one, two, three, or four objects present, and there could be any of four colors. Size was invariant, so that information value was completely controlled.
Table 1

Experiment I: Mean Latencies of Correct Responses (in Seconds)

\begin{tabular}{cccc} 
& \multicolumn{3}{c}{ Dimension Relevant } \\
\cline { 2 - 4 } $\begin{array}{c}\text { Adjective } \\
\text { Order }\end{array}$ & Color & Size & Either \\
\hline Normal & 1.44 & 1.46 & 1.46 \\
Inverted & 1.15 & 1.34 & 1.24 \\
\hline
\end{tabular}

The relative saliency appeared to be equal, although it was not objectively measured.

\section{Method}

\section{Subjects}

Twenty additional Ss with the same characteristics as those in Experiment I participated in this experiment.

\section{Materials}

The objects for this experiment were pencils, buttons, cups, fish, and leaves. All except the leaves were the small objects from Experiment I. The objects could be any of four colors-red, green, yellow, or brown-and could be one, two, three, or four in number.

The slide types for this experiment were identical to those in Experiment I, except that number was substituted for size. There were three types of experimental slides, all of which had two versions of the same object and two filler objects. For the color relevant slides, the two versions of the object were equal in number but differed in color. On the number relevant slides, the two objects were the same color but differed in quantity by two. that is, one and three objects or two and four. For the either relevant slides, the objects differed in both color and number. On each slide, the irrelevant filler objects were selected such that four different colors and four different numbers were always present (except where dictated by the experimental conditions). There were 10 each of the three experimental slide types. Filler slides were constructed as before. There were 25 neither relevant slides in which no object, color. or number was repeated. In the 25 both relevant slides, all four quadrants contained the same object represented by two levels of color and two levels of number, so that both adjectives were required for correct identification.

The referent was selected as in Experiment I, such that it occurred equally often in each of the quadrants. For the experimental slides, the other version of the referent occurred equally of ten in the other three quadrants.

The description of the referents were constructed exactly as in Experiment I, with half of each dimension relevant condition described by a normal order and half by an inverted order. A between-S variable was intonation pattern of the phrase, either stress or list. These were manipulated as before.

The slides were arbitrarily ordered in five blocks of 16 slides. consisting of two each of the three experimental conditions and five each of the two filler conditions. Within each block. no more than two criterion objects of the same color, two of the same number. one of the same object, two of the same adjective order, or two of the same dimension relevant could follow in succession.

Procedure

The procedure was identical to that in Experiment $I$.

\section{Results}

There were only 17 errors out of 480 responses. The 
Table 2

Experiment II: Mean Latencies of Correct Responses (in Seconds)

\begin{tabular}{llccc}
\hline \multirow{2}{*}{$\begin{array}{c}\text { Intona- } \\
\text { tion }\end{array}$} & $\begin{array}{c}\text { Adjective } \\
\text { Order }\end{array}$ & \multicolumn{3}{c}{ Dimension Relevant } \\
\cline { 3 - 5 } Stress & Normal & 1.53 & 1.13 & 0.97 \\
& Inverted & 1.21 & 1.50 & 1.11 \\
\multirow{2}{*}{ List } & Normal & 1.28 & 1.22 & 1.03 \\
& Inverted & 1.16 & 1.25 & 1.11 \\
Overall & Normal & 1.40 & 1.17 & 1.00 \\
& Inverted & 1.19 & 1.37 & 1.11 \\
\hline
\end{tabular}

errors were evenly distributed across conditions. A 2 by 2 by 3 analysis of variance was computed on the mean latencies for correct responses. The variables were intonation, adjective order, and dimension relevant. Dimension relevant was the only significant main effect $[F(2.36)=35.54, p<.001]$ : neither intonation nor adjective order approached significance. However, three interactions were significant: Dimension Relevant by Adjective Order $[\mathrm{F}(2.36)=31.75 . \mathrm{p}<.001]$, Dimension Relevant by Intonation $[F(2.36)=4.42 . p<.02]$, and the triple interaction of Dimension Relevant by Adjective Order by Intonation $[F(2.36)=12.21$. $p<.001]$. The mean latencies for each condition are presented in Table?.

First, consider the Dimension Relevant by Adjective Order interaction. jgnoring the interaction with intonation. The critical interaction for testing the main hypothesis was Color-Number Dimension Relevant by Adjective Order. This was clearly significant $[q(2,36)=13.80 . p<.001]$ : complete reversal in the response latencies was obtained. For number relevant slides, normal order of adjectives yielded faster responding than inverted order. and for color relevant slides an inverted order facilitated responding. In addition. in the either control condition, the normal adjective order produced faster identification than the inverted order $[q(2.36)=3.73, p<.02]$. Hence, the change of dimension from size to number was successful in removing the unpredicted results of Experiment I without vitiating the critical interaction.

Second. in order to interpret the triple interaction. let us relabel the conditions as to appropriateness of the adjective order for the dimension relevant. According to the pragmatic communication rule, the normal order was more appropriate in a number context and an inverted order was more appropriate in a color context. The appropriate conditions were those in which adjective order placed the relevant dimension first in the phrase. that is, color-inverted and number-normal. In these cases, intonation had t10 effect (.02-sec difference between stress and list intonation). The inappropriate conditions had adjective orders with the relevant dimension second. that is. color-normal and number-inverted. In these conditions. intonation had a powerful effect -stress intonation greatly lengthened the reaction time over a list presentation $(.25 \mathrm{sec})$. Intonation had no effect $(.03 \mathrm{sec})$ in the either condition. Thus, stressing the inappropriate adjective retarded the S's making either a color or a number identification, but stressing the appropriate adjective had no effect.

\section{DISCUSSION}

The results of both experiments suggest that the pragmatic communication rule is not only appropriate for the speaker as a production strategy but also for the listener as a comprehension strategy. When the adjective relevant to discriminating between two objects occurred first, responding was facilitated; otherwise, it was not. In the first experiment, the dimensions of color and size were not equated for either informativeness or saliency. An inverted order of adjectives unexpectedly facilitated responding in all contexts, not in just the color relevant. When the two dimensions were equated on informativeness and apparent saliency by replacing the size dimension with a number dimension, the results were consistent with the pragmatic communication rule. However, by replacing size with number, what was gained in control over the dimensions was lost in naturalness. Unlike the first experiment, in which phrases like the RED large car were acceptable with contrastive stress, when color and number are inverted, as in the RED two cars, the phrase sounds strange even with contrastive stress. If we accept the naturalness of the first experiment and the controls of the second, the results taken together do lend strong support for a listener's identification strategy in which context and adjective order interact.

The complicated effect of intonation pattern is a bit more difficult to interpret. One possible social function of the contrastive intonation by a speaker is to inform the listener that the speaker realizes the order of adjectives has been inverted. but the information would not actually facilitate identification by the listener. Hence. the lack of any effect in Experiment I was not unexpected. However. the relatively strong interaction of Intonation by Appropriateness in Experiment II vitiates such an interpretation. The only apparent difference between the two experiments was the substitution of number for size as the second adjective. A possible explanation. admittedly speculative. is that a listener expects the speaker to identify the relevant adjective with contrastive stress. especially with an inverted order. Hearing stress on the nonrelevant adjective initiates a nonfunctional comparison of that property. which in turn impedes the search for the relevant property.

The pragmatic communication rule proposed by Danks and Schwenk (1972) was stated as if the nonreferent context completely controls the ordering of adjectives. In the typical case. the nunreferents from which the referent is to be discriminated are not obvious 
or specifiable (Olson, 1970). In such situations, speakers prefer (Martin, 1969b; Danks \& Schwenk, 1972) and listeners are helped by (Experiment II) a "normal" adjective order. Hence, the question remains: How do we account for the normal ordering? We have suggested previously (Danks \& Glucksberg, 1971; Danks \& Schwenk, 1972) that the pragmatic communication rule does function in normal ordering but in a less specific fashion than in the experimental contexts used here. The pragmatic communication rule was applied here to specific adjectives in carefully controlled contexts. If some form of this rule is to describe normal adjective ordering, then the conditions for its use and the variables specified by the rule must be generalized.

First, the nonreferents that contrast with the referent are normally indefinite, unlike the controlled contexts of these experiments. Implied nonreferents that may be assumed by the listener are important for the speaker (Osgood, 1971). Thus, the context that influences the speaker's utterances is what he predicts is in the mind of the listener. Second, normal ordering refers to classes of adjectives, such as size and color, and their associated properties, rather than to specific adjectives that are associated with particular nouns and, hence, do not follow either the pragmatic communication rule or a normal ordering rule, for example, Swiss blue cheese. That certain adjective-noun combinations do not follow the rules of ordering has also been noted by Ertel (1971). Because these adjectives co-occur frequently with the particular nouns, they are relatively nondiscriminative and, hence, are not easily moved from their position adjacent to the noun.

The third and fourth points refer to an enlarged time frame for the influence of pragmatic factors on normal ordering. The model for these experiments implied that the pragmatic communication rule is applied on each trial by the $S$. In order to account for normal ordering, the pragmatic communication rule must be viewed in the context of the history of the language and the acquisition of language by the child. Classes of properties undoubtedly differ in their frequency of discriminating important events in the world. Consonant with the pragmatic communication rule, speakers would tend to order the adjectives with those frequently discriminating adjectives first and the less frequently discriminating adjectives last. As the language evolved, this order became regularized as the "normal" order. While we have been unable to find any evidence in the linguistic literature for or against this argument, Bever and Langendoen (1971) adduce diachronic evidence to support a similar argument that explains certain syntactic properties of English relative clauses. Fourth, an analogous argument can be formulated with respect to the child's acquisition of language. Those properties that are most important for organizing the child's experience will be coded earlier. Therefore, one would expect that adjective classes would, in general, be acquired in the order in which they occur prior to the noun. The more frequently a dimension (an adjective class) is used to discriminate objects from the universe, the sooner will the class become linguistically functional for the child. Thus, while the pragmatic communication rule is not claimed to determine normal adjective ordering each time a speaker uses two or more adjectives, the general communicative utility of the adjective class for specifying referents does control normal ordering indirectly.

\section{REFERENCES}

Bever. T. G., \& Langendoen, D. T. A dynamic model of the evolution of language. Linguistic Inquiry, 1971, 2, 433-463.

Danks, J. H., \& Glucksberg, S. Psychological scaling of adjective orders. Journal of Verbal Learning \& Verbal Behavior, 1971. 10, 63-67.

Danks, J. H., \& Schwenk, M. A. Prenominal adjective order and communication context. Journal of Verbal Learning \& Verbal Behavior, 1972, 11, 183-187.

Ertel, S. Pränominale adjektivfolgen and semantische tiefenstruktur. Studia Psychologica, 1971, 13, 127-137.

Martin, J. E. Some competence-process relationships in noun phrases with prenominal and postnominal adjectives. Journal of Verbal Learning \& Verbal Behavior. 1969a. 8. 471-480.

Martin, J. E. Semantic determinants of preferred adjective order. Journal of Verbal Learning \& Verbal Behavior, 1969b. 8. 697-704.

Oller, J. W., \& Sales, B. D. Conceptual restrictions on English: A psycholinguistic study. Lingua, 1969, 23. 209-232.

Olson, D. R. Language and thought: Aspects of a cognitive theory of semantics. Psychological Review, 1970. 77. 257-273.

Osgood, C. E. Where do sentences come from? In D. D. Steinberg and L. A. Jakobovitz (Eds.). Semantics. Cambridge: Cambridge University Press, 1971. Pp. 497-529.

Vendler, Z. Adjectives and nominalizations. The Hague: Mouton. 1968.

Winer, B. J. Statistical principles in experimental design. 12 nd ed.) New York: MoGraw-Hill, 1971

(Received for publication February 11. 1973; accepted May 15, 1973.) 